\title{
Expression of dual-specificity tyrosine-(Y)-phosphorylation- regulated kinase 2 (DYRK2) can be a favorable prognostic marker in pulmonary adenocarcinoma
}

\author{
Shin-ichi Yamashita, MD, Masao Chujo, MD, Keita Tokuishi, MD, Kentaro Anami, MD, \\ Michiyo Miyawaki, MD, Satoshi Yamamoto, MD, and Katsunobu Kawahara, MD
}

Objectives: We investigated the possibility of $D Y R K 2$, a dual-specificity tyrosine-(Y)-phosphorylation-regulated kinase gene, to predict survival for patients with pulmonary adenocarcinoma.

\begin{abstract}
Patients and Methods: One hundred forty-four patients with pulmonary adenocarcinoma underwent surgery in our institute from 2000 to 2008 . We used immunohistochemical analysis and real-time reverse-transcriptase polymerase chain reaction to determine the expression of DYRK2 and compared this with the clinicopathologic factors and survival.
\end{abstract}

\begin{abstract}
Results: We found no correlation between DYRK2 expression by immunohistochemical and clinicopathologic factors; however, a negative nodal status and negative lymphatic invasion were significantly associated with DYRK2 expression by reverse-transcriptase polymerase chain reaction. Five-year disease-free survival in the DYRK2-positive group $(75.4 \%)$ was significantly different from that in the negative group $(55.4 \% ; P=.03)$ by immunohistochemical analysis. The 5-year overall survival of $89.2 \%$ in the $D Y R K 2$-positive group was better than the $66.3 \%$ survival of the DYRK2-negative group $(P=.01)$. Quantitative real-time reverse-transcriptase polymerase chain reaction analyses showed a significant difference between positive and negative expressions for disease-free survival $(P=.003)$ and overall survival $(P=.007)$. In multivariate Cox regression analysis, negative $D Y R K 2$ protein and messenger RNA expression showed a worse prognostic value of survival (hazard ratio $[\mathrm{HR}]=4.7,95 \%$ confidence intervals $[\mathrm{CI}]=1.5-14.5, P=.007 ; \mathrm{HR}=2.5,95 \% \mathrm{CI}=1.1-6.1, P=.04$, respectively). When we analyzed adenocarcinoma cases except for bronchioloalveolar carcinoma, we found a close correlation between $D Y R K 2$ expression by immunohistochemical analysis and nodal status $(P=.03)$. Furthermore, disease-free survivals between positive and negative groups of DYRK2 expression by immunohistochemistry $(P=.03)$ and reverse-transcriptase polymerase chain reaction $(P=.02)$ without bronchioloalveolar carcinoma were significantly different. Overall survivals in both groups showed significant differences by immunohistochemistry $(P=.02)$ but not by reverse-transcriptase polymerase chain reaction $(P=.08)$.
\end{abstract}

Conclusions: These data showed that DYRK2 expression is associated with a favorable prognosis. (J Thorac Cardiovasc Surg 2009;138:1303-8)

Several clinical prognostic markers for lung cancer have been recognized to dichotomize the risk, such as age, sex, tumor stage, cellular differentiation, and vascular invasion. ${ }^{1}$ Furthermore, several molecular markers have been reported, such as epidermal growth factor receptor, ${ }^{2}$ a cell growth marker; $\mathrm{p} 53,{ }^{3}$ an apoptosis-related marker; and vascular endothelial growth factor, ${ }^{4}$ an angiogenesis-related marker. However, the clinical impact of these markers remains controversial and unclear.

\footnotetext{
From the Department of Surgery II, Faculty of Medicine, Oita University, Oita, Japan. Read at the Eighty-ninth Annual Meeting of The American Association for Thoracic Surgery, Boston, Massachusetts, May 9-13, 2009.

Received for publication May 1, 2009; revisions received July 16, 2009; accepted for publication Aug 7, 2009; available ahead of print Oct 12, 2009.

Address for reprints: Shin-ichi Yamashita, Department of Surgery II, Faculty of Medicine, Oita University, 1-1 Idaigaoka, Hasama, Yufu, Oita, 879-5593, Japan (E-mail: yamashi1@med.oita-u.ac.jp).

$0022-5223 / \$ 36.00$

Copyright $(C) 2009$ by The American Association for Thoracic Surgery

doi:10.1016/j.jtcvs.2009.08.003
}

DYRK2, a dual-specificity tyrosine-(Y)-phosphorylationregulated kinase gene, was identified as the most frequently amplified and overexpressed gene in lung adenocarcinoma. ${ }^{5}$ It was also previously reported that DYRK2 regulates $\mathrm{p} 53$ to induce apoptosis in response to DNA damage via the phosphorylation of Ser $45^{6}$; however, the functional significance of DYRK2 has not been reported and remains unclear. Recently, we identified the potential of DYRK2 as a predictor of chemotherapy benefit in non-small cell lung carcinoma (Anticancer Research, in press), and we found that bronchioloalveolar carcinoma (BAC), which shows better prognosis than the other adenocarcinoma subtypes, expressed a high amount of this protein.

We report our evaluation of this molecule and its ability to predict favorable survival.

\section{MATERIALS AND METHODS \\ Patients and Samples}

One hundred forty-four samples from patients treated surgically for lung adenocarcinoma were obtained at Oita University Hospital (Oita, Japan) 


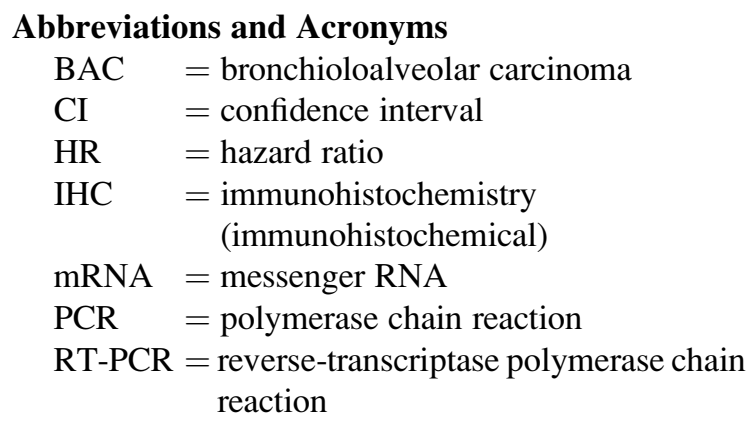

between 2000 and 2008. The study was approved by the Institutional Review Board of Oita University Hospital, and all patients completed informed consent forms. The samples were histologically diagnosed for primary adenocarcinoma of the lung by hematoxylin and eosin staining. None of the patients had received radiation therapy or chemotherapy before the operation. Adjacent normal lung tissue was also taken from all patients. Tissue specimens were frozen immediately with RNAlater (QIAGEN, Tokyo, Japan) and stored at $-80^{\circ} \mathrm{C}$ until RNA extraction. RNA from tissue samples was prepared with an RNeasy Mini Kit (QIAGEN).

\section{Immunohistochemical (IHC) Analysis}

Four-micrometer sections were prepared for tissue slides. Antigen was retrieved at $121{ }^{\circ} \mathrm{C}$ for 10 minutes in an autoclave with citrate buffer $(\mathrm{pH}$ 6.0) after deparaffinization. Ten percent goat serum (Nichirei, Tokyo, Japan) was used to block nonspecific binding. Staining with polyclonal anti-DYRK2 antibody (AP7534a; Abgent, San Diego, Calif) with diluents, 1:50, was performed overnight at $4{ }^{\circ} \mathrm{C}$. After a reaction with $3 \%$ hydrogen peroxide for 20 minutes at room temperature, polymer antirabbit (goat) antibody (K4002; Dako, Glostrup, Denmark) for DYRK2 was applied and incubated for 30 minutes at room temperature. Negative controls were incubated without the primary antibody.

IHC staining was evaluated as follows: 0 , no staining or faint cytoplasmic staining in less than $10 \%$ of tumor cells; $1+$, faint cytoplasmic staining in more than $10 \%$ of tumor cells; $2+$, weak or moderate cytoplasmic staining in more than $10 \%$ of tumor cells; $3+$, more than $10 \%$ of strong cytoplasmic staining. Since the DYRK2 IHC staining study has not been reported previously, 0 or $1+$ staining intensity was considered DYRK 2 negative and $2+$ or $3+$ staining was considered positive according to a previous report referring to human epidermal growth receptor- 2 protein. ${ }^{7}$ For evaluation reliability, two independent assessors estimated the staining positivity of two serial sections.

\section{Quantitative Polymerase Chain Reaction (PCR) Analysis}

For quantitative evaluation of the RNA expression by PCR, we used Taqman PCR methods (TaqMan Gene Expression Assays; Applied Biosystems, Tokyo, Japan) as previously reported. ${ }^{8}$ The DYRK2 gene was amplified by the following primer set: forward: gccatgttaaccaggaaacc, reverse: gaagcctgaagctgacgaac.

The DYRK2 gene internal probe was the universal probe library probe No. 70 (Roche, Mannheim, Germany). The PCR amplification condition was 1 cycle at $50{ }^{\circ} \mathrm{C}$ for 2 minutes and $95^{\circ} \mathrm{C}$ for 10 minutes followed by 50 cycles at $95{ }^{\circ} \mathrm{C}$ for 15 seconds and $60^{\circ} \mathrm{C}$ for 1 minute. The measured value was calculated by comparative threshold cycle methods ${ }^{8}$ and glyceraldehyde-3-phosphate dehydrogenase gene amplification was used as a control. All reactions were duplicated. The amounts of DYRK2 messenger RNA (mRNA) were expressed as n-fold glyceraldehyde-3-phosphate dehydrogenase mRNA and the levels were compared relative to the normal lung tissues pool. A cutoff value of DYRK2 mRNA expression greater than 2.7, which was the value of normal sample pool, was identified as a positive expression and lower as a negative expression.

\section{Statistical Analysis}

All statistical analysis was performed with Stat View J5.0 (SAS Institute, Inc, Tokyo, Japan). The different variables of the tumors and normal tissues were analyzed with $\chi^{2}$ tests or Fisher's exact tests. Time to progression was analyzed by the Kaplan-Meier method and evaluated by the logrank test.

\section{RESULTS \\ Relationship Between Clinicopathologic Characteristics and DYRK2 Expression}

We investigated the relationship between the clinicopathologic characteristics of adenocarcinoma and DYRK2 expression. As shown in Table 1, we found no correlation between $D Y R K 2$ expression by IHC and any clinical factors; however, DYRK2 mRNA expression in node-negative or negative lymphatic invasion was significantly higher than in node-positive or positive lymphatic invasion by reversetranscriptase polymerase chain reaction (RT-PCR). The expression pattern of DYRK2 is shown in Figure 1. Positive cases showed strong granular staining in the cytoplasm of cancer cells from the resected specimen of lung adenocarcinoma (Figure 1).

\section{Prognostic Values of DYRK2}

Figure 2 shows disease-free survival and overall survival according to the stratification of DYRK2 expression. Fiveyear disease-free survival in the DYRK2-positive group (75.4\%) was significantly different from that in the negative group $(55.4 \% ; P=.03$, Figure $2, A)$ by IHC. The 5 -year overall survival was $89.2 \%$ in the $D Y R K 2$-positive group as compared with $66.3 \%$ in the DYRK2-negative group $(P=.01$, Figure $2, B)$. Quantitative real-time RT-PCR analyses showed a significant difference between positive expression and negative for disease-free survival (Figure 2, $C ; P=.003$ ) and overall survival (Figure $2, D ; P=.007$ ).

In multivariate Cox regression analysis, negative DYRK2 protein expression indicated a worse prognosis of survival (hazard ratio $[\mathrm{HR}]=4.7,95 \%$ confidence interval $[\mathrm{CI}]=$ $1.5-14.5 ; P=.007$, Table 2 ), in addition to tumor size. Furthermore, the potential of a prognostic factor by mRNA expression of DYRK2 was elucidated by multivariate Cox regression analysis (Table 3). Negative DYRK2 expression showed a significantly higher hazard ratio $(\mathrm{HR}=2.5$, $95 \% \mathrm{CI}=1.1-6.1 ; P=.04)$, the same as tumor size.

When we analyzed adenocarcinoma cases except the $\mathrm{BAC}$, we found the close correlation between DYRK2 expression by IHC and nodal status $(P=.03)$, but no correlation between them by RT-PCR $(p=.07)$. Furthermore, we found that disease-free survivals between the positive and negative groups of DYRK2 expression by IHC and RTPCR without BAC were significantly different (Figure 3, 
TABLE 1. Patient characteristics

\begin{tabular}{|c|c|c|c|c|c|}
\hline Characteristics & $\begin{array}{c}\text { All } \\
\text { patients }\end{array}$ & $\begin{array}{c}\text { DYRK2 } \\
(\%) \text { IHC } \\
\text { positive }\end{array}$ & $\begin{array}{c}P \\
\text { value } \\
\end{array}$ & $\begin{array}{c}\text { DYRK2 } \\
(\%) \text { RT-PCR } \\
\text { positive } \\
\end{array}$ & $\begin{array}{c}P \\
\text { value } \\
\end{array}$ \\
\hline \multicolumn{6}{|l|}{ Age } \\
\hline$<70$ & 80 & $35(44)$ & .57 & $49(61)$ & .12 \\
\hline$\geq 70$ & 64 & $25(39)$ & & $31(48)$ & \\
\hline \multicolumn{6}{|l|}{ Sex } \\
\hline Male & 70 & $28(40)$ & .69 & $35(50)$ & .19 \\
\hline Female & 74 & $32(43)$ & & $45(61)$ & \\
\hline \multicolumn{6}{|l|}{ Histologic subtype } \\
\hline Ad & 102 & $43(42)$ & .85 & $50(49)$ & .01 \\
\hline $\mathrm{BAC}$ & 42 & $17(40)$ & & $30(71)$ & \\
\hline \multicolumn{6}{|l|}{ Tumor size } \\
\hline$<30$ & 92 & $37(40)$ & .06 & $55(60)$ & .17 \\
\hline$\geq 30$ & 52 & $22(52)$ & & $25(60)$ & \\
\hline \multicolumn{6}{|l|}{ Nodal status } \\
\hline Positive & 112 & $51(46)$ & .08 & $69(62)$ & .006 \\
\hline Negative & 32 & $9(28)$ & & $11(34)$ & \\
\hline \multicolumn{6}{|l|}{ Vascular invasion } \\
\hline Positive & 102 & $46(45)$ & .19 & $61(60)$ & .11 \\
\hline Negative & 42 & $14(33)$ & & $19(45)$ & \\
\hline \multicolumn{6}{|l|}{ Lymphatic invasion } \\
\hline Positive & 104 & $45(43)$ & .52 & $64(62)$ & .02 \\
\hline Negative & 40 & $15(38)$ & & $16(40)$ & \\
\hline Stage & & & .43 & & .1 \\
\hline IA & 72 & $32(44)$ & & $47(64)$ & \\
\hline IB & 27 & $13(48)$ & & $14(52)$ & \\
\hline IIA & 5 & $1(20)$ & & $0(0)$ & \\
\hline IIB & 6 & $1(17)$ & & $2(33)$ & \\
\hline IIIA & 16 & $4(25)$ & & $8(50)$ & \\
\hline IIIB & 13 & $7(54)$ & & $6(46)$ & \\
\hline IV & 4 & $2(50)$ & & $3(75)$ & \\
\hline
\end{tabular}

$I H C$, Immunohistochemistry; $R T-P C R$, reverse-transcriptase polymerase chain reaction; $A d$, adenocarcinoma; $B A C$, bronchioloalveolar carcinoma.

$A$ and $C$ ). Overall survivals in both groups showed significant differences by IHC (Figure 3, B), but not by RT-PCR (Figure 3,D). Taken together, adenocarcinoma expressing $D Y R K 2$ showed better prognosis, and this protein can be a favorable prognostic marker despite excluding BAC.

\section{DISCUSSION}

In this study, we focused on the assessment of DYRK2 as new prognostic markers of lung adenocarcinoma. DYRK2 is a dual-specificity tyrosine-(Y)-phosphorylation- regulated kinase gene, which has the potential to phosphorylate both Ser/Thr and Tyr. ${ }^{5,9}$ DYRK families are involved in regulating processes such as cell proliferation, cytokinesis, and cell differentiation. Furthermore, it is known that DYRK2 is an effector kinase for Ser46 of p53, which leads to apoptosis in the response to severe DNA damage. ${ }^{6}$ As the knock down of the DYRK2 function attenuates doxorubicin (Adriamycin)-induced apoptosis, $D Y R K 2$ has a key role in p53-induced apoptosis. In addition, DYRK2 can induce apoptosis in a p53-independent manner. ${ }^{6}$ When we investigated the relationship between $D Y R K 2$ expression and $\mathrm{p} 53$ expression by IHC, the p53 positivity was quite low $(37.5 \%, 15 / 40$ cases) and no relationships existed between p53 and DYRK2 expression (Anticancer Research, in press). Therefore, we did not check p53 expression in this study, and DYRK2 may exert independent manner of p53 function. Further, we failed to show the correlation between DYRK2 expression and phosphor-p53 by anit-phospho-p53 (S46) antibody, because DYRK2 functions in response to the DNA damaging stimuli.

DYRK2 is overexpressed and amplified in lung adenocarcinoma and esophageal carcinomas ${ }^{5}$; however, the role of $D Y R K 2$ in lung adenocarcinoma remains unclear. We found that $D Y R K 2$ expression was associated with a better prognosis in lung adenocarcinoma. Additionally, the relationship between node positivity and DYRK2 expression was conversely related; therefore, patients with $D Y R K 2$-positive tumors might show better prognosis than those with negative tumors. As previously reported, ${ }^{9} D Y R K 2$ is associated with cell cycle regulation, proliferation, and differentiation. Our results may support these functions in lung adenocarcinoma. In the present study, we found $40.6 \%$ of DYRK2-positive cases by IHC and $56.1 \%$ by RT-PCR, higher than in previous reports. ${ }^{5}$ These differences might be due to the staining methods, use of a different antibody, or the
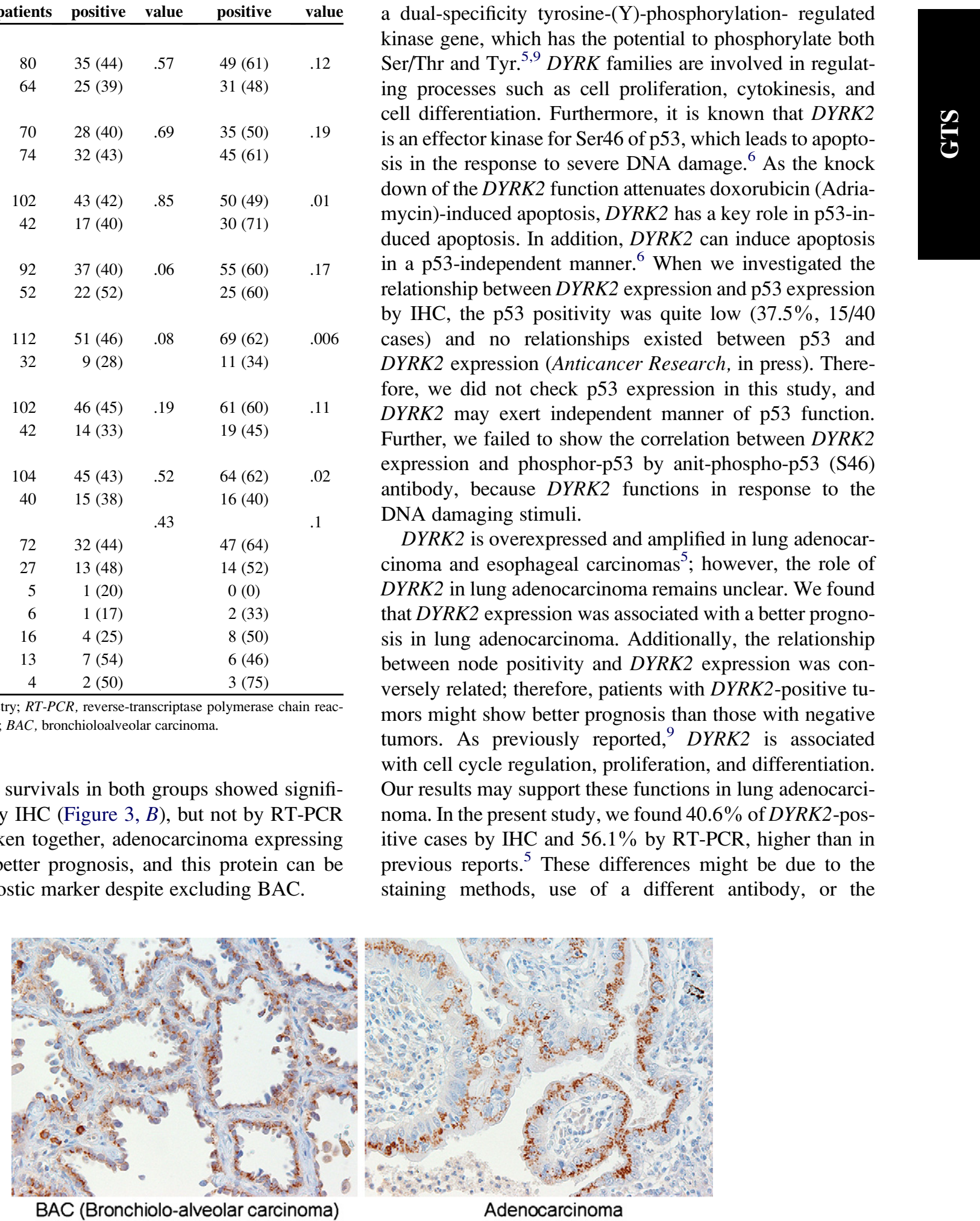

FIGURE 1. Representative DYRK2 protein expression in adenocarcinoma and bronchioloalveolar carcinoma by immunohistochemistry. Cytoplasm of cancer cells was stained strongly in $3+$ cases $(400 \times)$. 

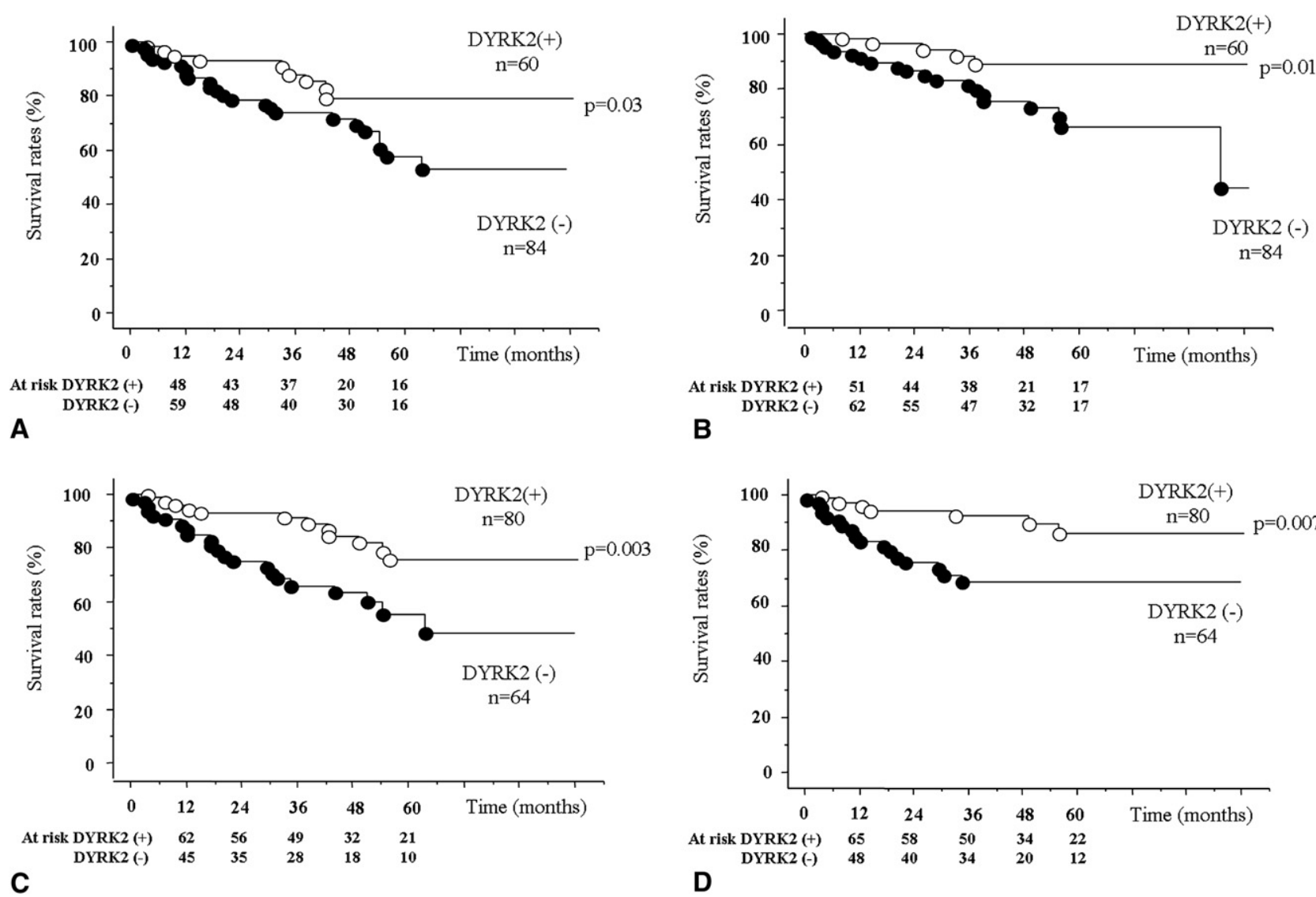

FIGURE 2. Disease-free survival (A) and overall survival (B) according to $D Y R K 2$ protein expression. Five-year disease-free survival is $75.4 \%$ in $D Y R K 2$ positive group and $55.4 \%$ in negative $(P=.03)$. Five-year overall survival is $85.4 \%$ in $D Y R K 2$-positive group and $68.5 \%$ in negative $(P=.01)$. These are all statistically significant. Disease-free survival (C) and overall survival (D) according to $D Y R K 2 \mathrm{mRNA}$ expression. Five-year disease-free survival is $75.4 \%$ in DYRK2-positive group and $55.4 \%$ in negative $(P=.003)$. Five-year overall survival is $89.2 \%$ in $D Y R K 2$-positive group and $66.3 \%$ in negative $(P=.007)$. These are all statistically significant.

evaluation of IHC and sensitivity of RT-PCR. We could not deny the overestimation owing to the possibility of artifacts. Since the concordance rate between IHC positivity and mRNA overexpression by real-time RT-PCR was $62.5 \%$, posttranscriptional modification of this gene expression may exist. Inasmuch as both mRNA and protein expression study showed a significant correlation between DYRK2 and prognosis in lung adenocarcinoma, our result may be more plausible than previous reports.

TABLE 2. Multivariate Cox proportional hazard model

\begin{tabular}{llll}
\hline \multicolumn{1}{c}{ Characteristics } & HR & $\mathbf{9 5} \%$ CI & $\boldsymbol{P}$ value \\
\hline Histologic subtype (BAC vs Ad) & 3.6 & $0.4-30.7$ & .23 \\
Tumor size $(<30 \mathrm{~mm}$ vs $\geq 30 \mathrm{~mm})$ & 3.1 & $1.2-7.9$ & .02 \\
Nodal status (negative vs positive) & 1.6 & $0.6-4.5$ & .36 \\
Vascular invasion (negative vs positive) & 1.3 & $0.5-3.3$ & .55 \\
Lymphatic invasion (negative vs positive) & 2.2 & $0.7-6.9$ & .16 \\
DYRK2 expression (IHC) (positive vs negative) & 4.7 & $1.5-14.5$ & .007 \\
\hline HR, Hazard ratio; $C I$, confidence interval; $B A C$, bronchioloalveolar carcinoma; $A d$, \\
adenocarcinoma; $I H C$, immunohistochemistry.
\end{tabular}

Although DYRK2 is not expressed in normal lung epithelium and advanced acinar or papillary adenocarcinoma cells, BAC, which arose from type II alveolar cells, ${ }^{10}$ and adenocarcinoma with a BAC component expressed higher levels of DYRK2, but the difference was not significant. Inasmuch as BAC possesses relatively favorable behavior, ${ }^{11}$ a positive DYRK2 expression might lead to a better prognosis than negative expression. Not only the BAC subtype but also

TABLE 3. Multivariate Cox proportional hazard model

\begin{tabular}{lclc}
\hline \multicolumn{1}{c}{ Characteristics } & HR & $\mathbf{9 5} \%$ CI & $\boldsymbol{P}$ value \\
\hline Histologic subtype (BAC vs Ad) & 3.9 & $0.5-31.8$ & .2 \\
Tumor size (<30 mm vs $\geq 30 \mathrm{~mm})$ & 2.6 & $1.1-6.4$ & .03 \\
Nodal status (negative vs positive) & 1.9 & $0.7-5.2$ & .22 \\
Vascular invasion (negative vs positive) & 1.3 & $0.5-3.3$ & .56 \\
Lymphatic invasion (negative vs positive) & 1.6 & $0.5-4.6$ & .41 \\
DYRK2 expression (RT-PCR) (positive vs & 2.5 & $1.1-6.1$ & .04
\end{tabular}
negative)

$H R$, Hazard ratio; $C I$, confidence interval; $B A C$, bronchioloalveolar carcinoma; $A d$, adenocarcinoma; $R T-P C R$, reverse transcription polymerase chain reaction. 

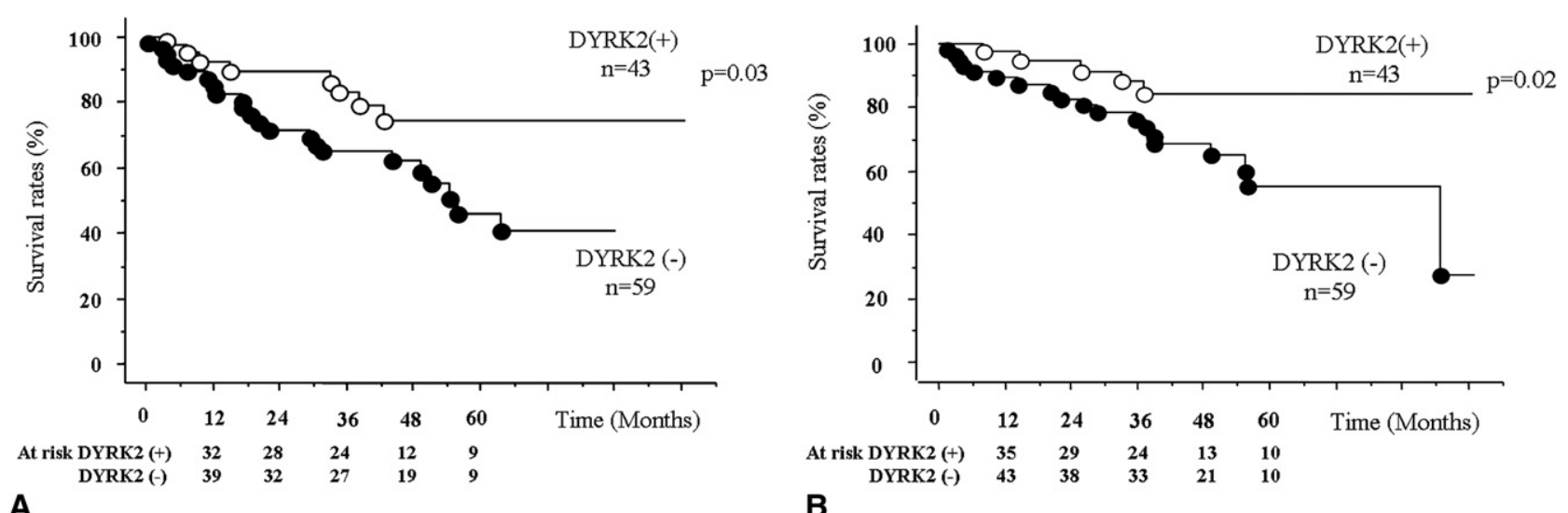

A

B
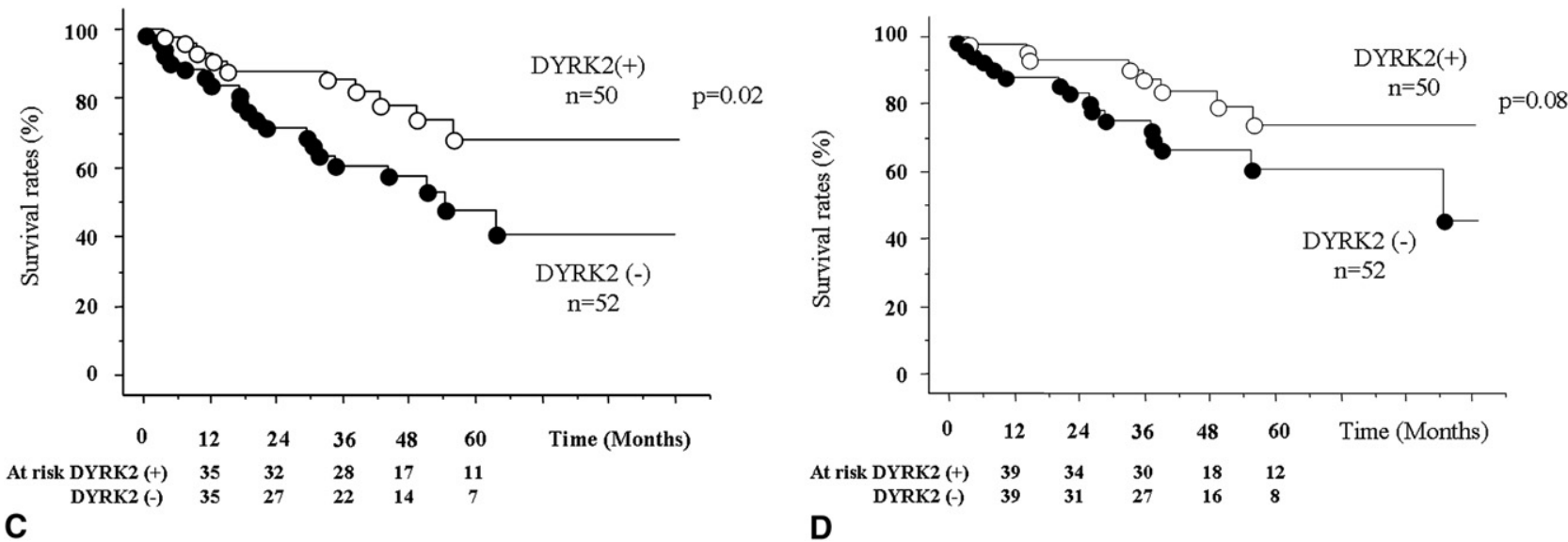

FIGURE 3. Disease-free survival (A) and overall survival (B) according to DYRK2 protein expression in adenocarcinoma except bronchioloalveolar carcinoma. Five-year disease-free survival is $74.5 \%$ in $D Y R K 2$-positive group and $45.9 \%$ in negative $(P=.03)$. Five-year overall survival is $84.3 \%$ in DYRK2-positive group and $55.1 \%$ in negative $(P=.02)$. These are all statistically significant. Disease-free survival $(C)$ and overall survival $(D)$ according to DYRK2 mRNA expression in adenocarcinoma except bronchioloalveolar carcinoma. Five-year disease-free survival is $67.9 \%$ in $D Y R K 2$-positive group and $47.7 \%$ in negative $(P=.02)$. Five-year overall survival is $73.6 \%$ in $D Y R K 2$-positive group and $60.3 \%$ in negative $(P=.08)$, but not statistically significant.

DYRK2-positive adenocarcinoma showed a better prognosis. Taken together, DYRK2 itself contributes to a better prognosis and might be related to the slow growth of tumor cells. Although the understanding of DYRK2 expression in BAC is unclear, it may play a critical role in the initiation of carcinogenesis because of the negative expression in normal lung tissues and advanced adenocarcinoma. We researched the function of DYRK2 in adenocarcinoma and found that adenocarcinoma with a BAC component had high expression levels of this protein or mRNA (not significant). These findings led to the speculation that DYRK2 is related to early events in carcinogenesis and is a powerful tool to distinguish BAC from adenocarcinoma. Furthermore, the sequence from BAC to adenocarcinoma might be elucidated by this molecule.

In conclusion, our study demonstrated that patients with DYRK2-positive adenocarcinoma had a better prognosis than patients with DYRK2-negative adenocarcinoma. $D Y R K 2$ might therefore be a powerful tool to stratify favor- able candidate groups in lung cancer patients. Further investigation of DYRK2 might offer new insight into this possibility.

We appreciate the technical support of Ms Yoko Miyanari from the Department of Surgery II, Oita University Faculty of Medicine.

\section{References}

1. Mountain CF. Revisions in the International System for Staging Lung Cancer. Chest. 1997;111:1710-7.

2. Brabender J, Danenberg KD, Metzger R, Schneider PM, Park J, Salonga D, et al. Epidermal growth factor receptor and HER2-neu mRNA expression in non-small cell lung cancer is correlated with survival. Clin Cancer Res. 2001;7:1850-5.

3. Tsao MS, Aviel-Ronen S, Ding K, Lau D, Liu N, Sakurada A, et al. Prognostic and predictive importance of p53 and RAS for adjuvant chemotherapy in non small-cell lung cancer. J Clin Oncol. 2007;25:5240-7.

4. Ogawa E, Takenaka K, Yanagihara K, Kurozumi M, Manabe T, Wada H, et al. Clinical significance of VEGF-C status in tumour cells and stromal macrophages in non-small cell lung cancer patients. Br J Cancer. 2004;91:498-503.

5. Miller CT, Aggarwal S, Lin TK, Dagenais SL, Contreras JI, Orringer MB, et al Amplification and overexpression of the dual-specificity tyrosine-(Y)- phosphorylation regulated kinase 2 (DYRK2) gene in esophageal and lung adenocarcinoma. Cancer Res. 2003;63:4136-43. 
6. Taira N, Nihira K, Yamaguchi T, Miki Y, Yoshida K. DYRK2 is targeted to the nucleus and controls p53 via Ser46 phosphorylation in the apoptotic response to DNA damage. Mol Cell. 2007;25:794-6.

7. Pothos A, Plastira K, Plastiras A, Vlachodimitropoulos D, Goutas N, Angelopoulou R. Comparison of chromogenic in situ hybridisation with fluorescence in situ hybridisation and immunohistochemistry for the assessment of her-2/ neu oncogene in archival material of breast carcinoma. Acta Histochem Cytochem. 2008;41:59-64.

8. Aarskog NK, Vedeler CA. Real-time quantitative polymerase chain reaction. A new method that detects both the peripheral myelin protein 22 duplication in CharcotMarie-Tooth type $1 \mathrm{~A}$ disease and the peripheral myelin protein 22 deletion in hereditary neuropathy with liability to pressure palsies. Hum Genet. 2000;107:494-8.

9. Yoshida K. Role for DYRK family kinases on regulation of apoptosis. Biochem Pharmacol. 2008;76:1389-94.

10. Kim CF, Jackson EL, Woolfenden AE, Lawrence S, Babar I, Vogel S, et al. Identification of bronchioalveolar stem cells in normal lung and lung cancer. Cell. 2005; 121:823-35.

11. Aviel-Ronen S, Coe BP, Lau SK, da Cunha Santos G, Zhu CQ, Strumpf D, et al. Genomic markers for malignant progression in pulmonary adenocarcinoma with bronchioloalveolar features. Proc Natl Acad Sci U S A. 2008;105:10155-60.

\section{Discussion}

Dr David M. Jablons (San Francisco, Calif). I have 3 questions. When you did RT-PCR, what statistical method did you use to validate the cutoff, and how did you choose a cutoff for an arbitrary positive or negative value of 2.7 ?

Dr Yamashita. I used the cutoff point by using the estimation of normal samples, because I always measured the expression of DYRK2 by using normal expression samples, messenger expression. The cutoff point is 2.7, and more than 2.7 of DYRK2 expression is positive.

Dr Jablons. Did you do that by a training set and a validation?

Dr Yamashita. No. We have to do that.

Dr Jablons. Second, have you looked at patients with more advanced stage non-small cell lung cancer to see whether this gene actually has any prognostic and/or even predictive value, especially in relationship to alleged sensitivity to a platinum-based doublet chemotherapy?

Dr Yamashita. These samples were obtained from stage I to stage IIIA. I haven't checked stage IV or more aggressive tumors.

Dr Jablons. Was this gene predictive in patients with stage IIIA disease?

Dr Yamashita. Unfortunately, I estimated the expression of IIIA in the small samples. However, in our analysis, tumor size and the DYRK2 expression are prognostic factors. I think that IIIA is more-no, I or II has a depressed expression of DYRK2. Maybe IIIA or IV should be downregulated of DYRK2. I'm not sure, so I have to check by staging.

Dr Jablons. One last question: You mentioned in the manuscript that BAC tumors had a higher expression. Do you think that your prognostic value is represented by the fact that you are picking up more BAC tumors in the stage I patient population?

Dr Yamashita. Not yet. That is an important question. I will check the DYRK2 expression in BAC. So I have to check the prognosis according to the DYRK2 expression in BAC. 